Article

\title{
A Study on the Control Performance of Electronic Differential System for Four-Wheel Drive Electric Vehicles
}

\author{
Dejun Yin ${ }^{1}$, Danfeng Shan ${ }^{1}$ and Jia-Sheng $\mathrm{Hu}^{2, *}$ \\ 1 School of Mechanical Engineering, Nanjing University of Science and Technology, Nanjing 210094, China; \\ yin@njust.edu.cn (D.Y.); shandanfeng@foxmail.com (D.S.) \\ 2 Department of Greenergy, National University of Tainan, No. 33, Sec. 2, Shu-Lin St., Tainan 700, Taiwan \\ * Correspondence: jogson@ieee.org; Tel.: +886-6-260-5051
}

Academic Editor: Shoou-Jinn Chang

Received: 29 September 2016; Accepted: 9 January 2017; Published: 12 January 2017

\begin{abstract}
The electronic differential system (EDS) is an important issue for four-wheel drive electric vehicles. This paper delineates an advanced EDS steering strategy and carries out a careful study of its control performance by numerical simulations that comply with the requirements of ISO4238:2012. The results demonstrate that the EDS feedback gain plays an important role to its control performance, particularly to its steering characteristics. Moreover, the analysis and discussion disclose the mechanism of the relationship between the feedback gain and the steering characteristics, which will contribute to further research and EDS development.
\end{abstract}

Keywords: electronic differential system; ISO4138:2012; four-wheel drive electric vehicle

\section{Introduction}

Most automotive differentials use limited slip differential (LSD) to turn a car. Under normal circumstances, LSD can follow the normal steering curvature at high speeds. However, if the vehicle is driven at low speeds, it will deviate from the original trajectory due to the reduction in traction. Cornering is a problem in LSD that can lead to vehicles not traveling in one direction and it, as shown in Figure 1, can be categorized into three types: under-steering, neutral-steering, and over-steering. Under everyday skidding scenarios, these driving styles are adjustable by the electronic differential system (EDS) [1-7]. The EDS aims to distribute the torque command to the left and right wheels for the required vehicle motion and orientation control. These two input commands are initiated so that we can easily pilot the vehicle for moving forward and orientation control via the steering wheel and pedal (i.e., two decoupling commands). Hence, different EDS settings represent different driving styles. For example, in the under-steering EDS setting, the vehicle continues to travel in one direction despite sharply turning the steering wheel. Conversely, in over-steering EDS, the vehicle rotates more than requested by the driver, causing the rear of the vehicle to swerve outwards. Different EDS settings respond to different steering experiences and energy consumptions differently. In order for vehicles to travel in the original trajectory at any speed and not slip, we plan to employ a new EDS to improve the disadvantages of LSD.

This paper presents EDS system analysis, modeling, and a simulation of a four-wheel drive electric vehicle, which is equipped by four independent in-wheel motors on four propulsion wheels. For example, in-wheel motor electric vehicles have independently equipped motors that drive each wheel [8-10]. EDS is needed for each electric vehicle with decentralized direct drive. This study proposes novel EDS control based on a feedback adjustment, which is investigated under the ISO4138:2012 standard [11]. Under different feedback gain $K$, the steering characteristics can be adjusted. Torque distribution can be treated as a central problem of large power vehicles. Because 
the distance between the transmission of the left and right propulsion wheels is unequal, it makes torque output inhomogeneous, resulting in a slight slip during acceleration. Basically, the designated feedback gain $K$ can solve the torque and power distribution of specific cornering conditions, a mechanism which will be discussed in the following sections. Moreover, this study presents its relevant theoretical foundations.

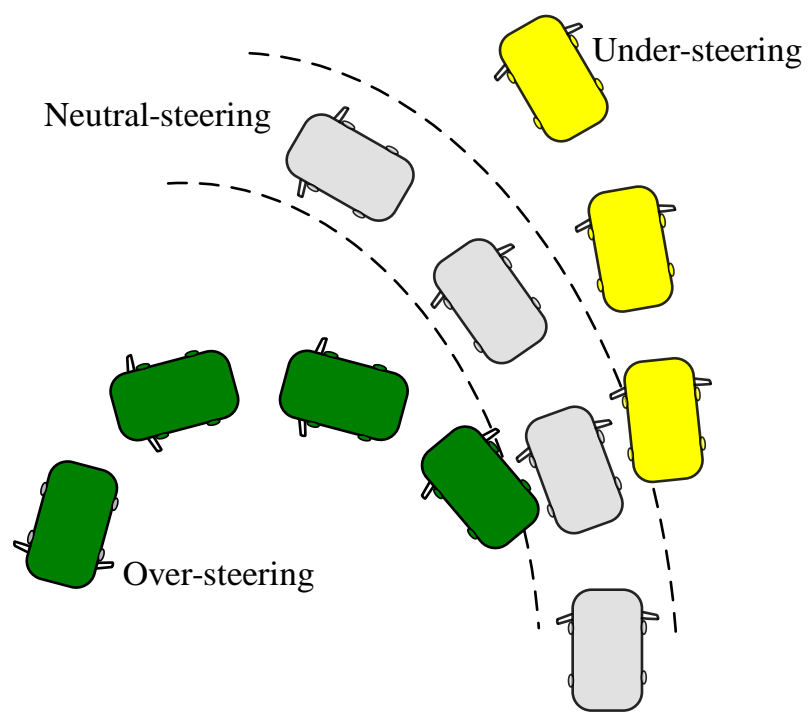

Figure 1. Steering responses while taking a curve.

\section{Modeling of EDS}

EDS was created to solve the turning issue for four-wheel drive electric vehicles. Many successful studies have since been proposed [12-16]. Figure 2 shows a basic scenario of a turning vehicle. It shows the kinematic model of the proposed system in a left turning maneuver, where $\delta$ is the turning angle command, $\delta_{1}$ and $\delta_{2}$ are the turning angle of the front left and right wheels, $L$ is the wheelbase, $D$ is the rear gauge, $\omega_{\delta}$ is the rotational speed of turning, $R$ is the radius of turning, $V$ is vehicle speed, $V_{1}$, $V_{3}$ are the tangential speed of the front and rear left wheels, and $V_{2}, V_{4}$ are the tangential speed of the front and rear right wheels. Generally, the speed of both the front and rear wheels should be the same in order to keep the vehicle traveling in a straight track. However, if the vehicle makes a turn, the speed differential must be constructed by a mechanism that entails less wire slipping and scrapping. Clearly, for a left turn scenario, if $\omega_{0}$ is the preferred rotating command, then $V=\omega_{0} R$ and

$$
\begin{aligned}
& V_{1}=V\left(1-\frac{D}{R}\right) \\
& V_{2}=V\left(1+\frac{D}{R}\right) \\
& V_{3}=V\left(\frac{R-\frac{D}{2}}{R}\right) \\
& V_{4}=V\left(\frac{R+\frac{D}{2}}{R}\right)
\end{aligned}
$$

where the radius of the turning curvature can be found as

$$
R=\frac{L}{\tan \delta} .
$$




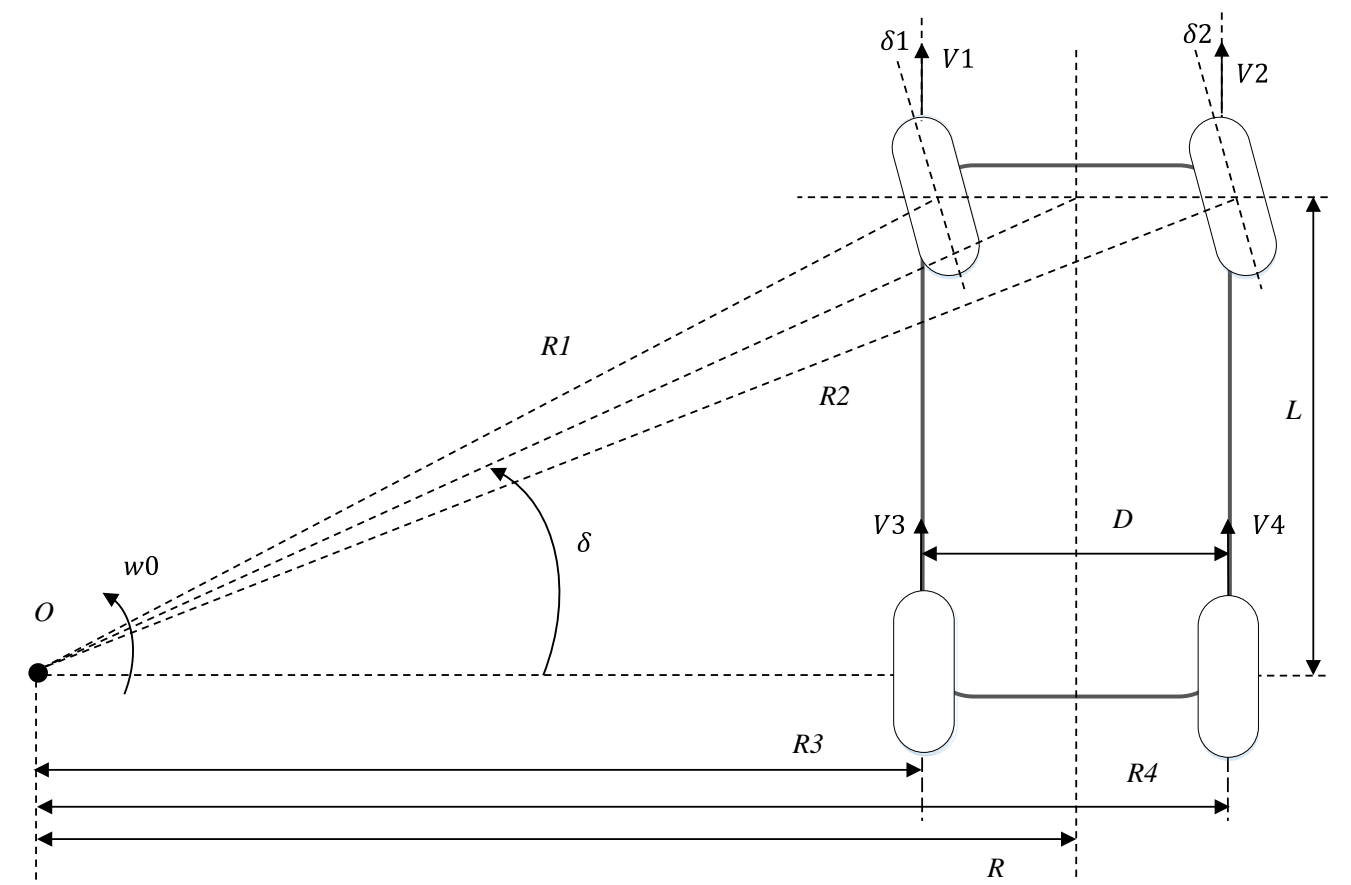

Figure 2. Kinematic model of proposed system in left turning maneuver.

The aforementioned studies solve the EDS for speed control in a four-wheel drive vehicle. However, usually the vehicle is operated by users under a pedal command (acceleration command) for torque distribution. Consequently, the aforementioned approaches are not so straightforward. Figure 3 shows the scheme of the proposed EDS. Figure 4 illustrates its corresponding control block diagram. Clearly, the actual vehicle speed is set as follows considering cost-effectiveness:

$$
V=\frac{V_{3}+V_{4}}{2}
$$

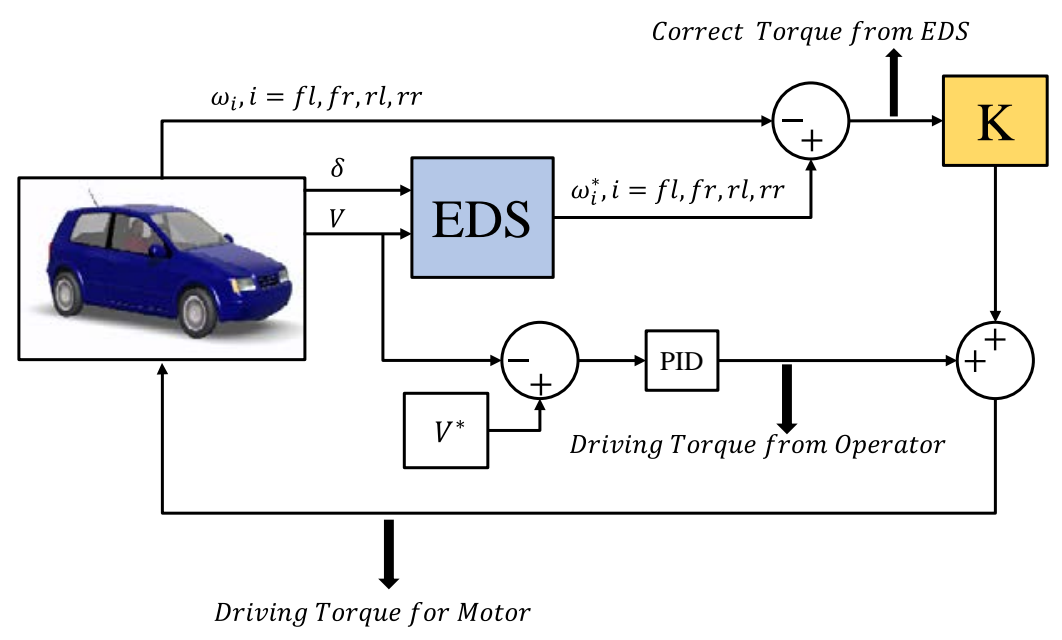

Figure 3. Proposed electronic differential system (EDS). 


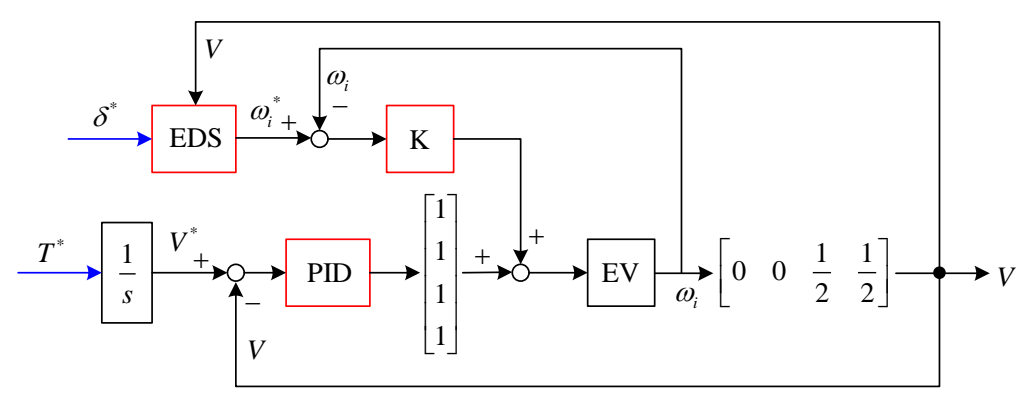

Figure 4. Control block diagram of the proposed system.

For acquiring the vehicle speed in practice without expensive sensors, studies $[17,18]$ provide solutions. Figure 5 shows the $K$ gain adjusting philosophy. Basically, sufficient $K$ gain falls into an acceptable region as

$$
K_{\min }^{*}<K<K_{\max }^{*} .
$$

Note that the vehicle pretends to over-steer if the $K$ gain is larger than $K_{\max }^{*}$. Hence, the steering angle will become sensitive and cause the turning radius to get smaller. Finally, the speed difference between the left and right wheels increases. Conversely, if the $K$ gain is smaller than $K_{\mathrm{min}^{*}}^{*}$ the steering angle will become insensitive and cause the turning radius to decrease. Consequently, the speed difference between the left and right wheels also decreases.

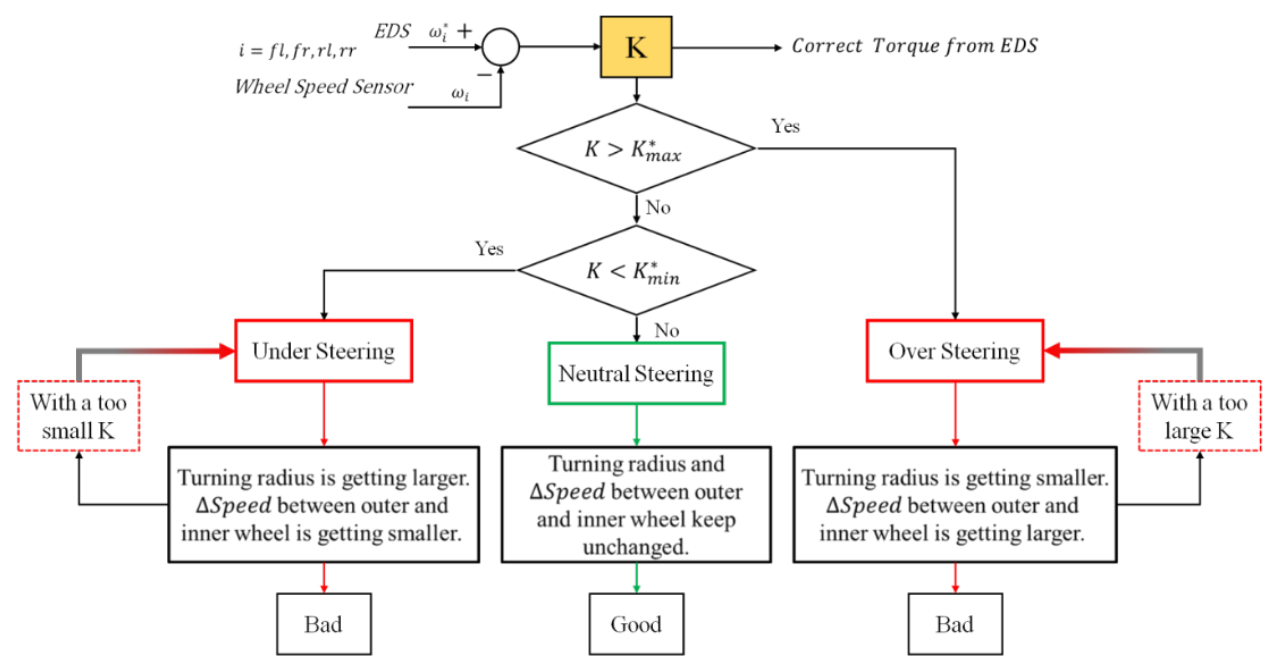

Figure 5. Philsophy of $K$ gain adjusting.

\section{Simulation Setup}

In order to evaluate the performance of the proposed approach, this study applied CarSim software (8.03, Mechanical Simulation, Ann Arbor, MI, USA) with Simulink (R2013b, MathWorks, Natick, MA, USA) to carry out the simulation. Table 1 shows the specifications of the test vehicle.

Table 1. Vehicle specification.

\begin{tabular}{cc}
\hline Sprung Mass & $\mathbf{1 5 2 7} \mathbf{~ k g}$ \\
\hline Unsprung mass & $182 \mathrm{~kg}$ \\
Width $D$ & $1535 \mathrm{~mm}$ \\
Height of center of gravity & $540 \mathrm{~mm}$ \\
Wheelbase $L$ & $2690 \mathrm{~mm}$ \\
Unloaded wheel radius & $394 \mathrm{~mm}$ \\
\hline
\end{tabular}


To probe the EDS performance, ISO4138:2012 suggests three methods: (1) constant radius; (2) constant steering-wheel angle; and (3) constant speed. In this paper, the radius of the circular orbit is set to $30 \mathrm{~m}$, lateral acceleration is $0.3 \mathrm{~g}$, the coefficient of friction of the road is 0.8 , and the simulation steering angle (front wheel) is $0.16 \mathrm{rad}$. Hence, the vehicle is steered under a constant speed of $33.8 \mathrm{~km} / \mathrm{h}$. Figure $6 \mathrm{a}$ shows the command of the front steering-wheel angle. Figure $6 \mathrm{~b}$ illustrates the desired circular orbit from neutral steering. The proportional-integral-derivative controller (PID controller) gains for velocity controller are set as $250,15,10$, respectively.

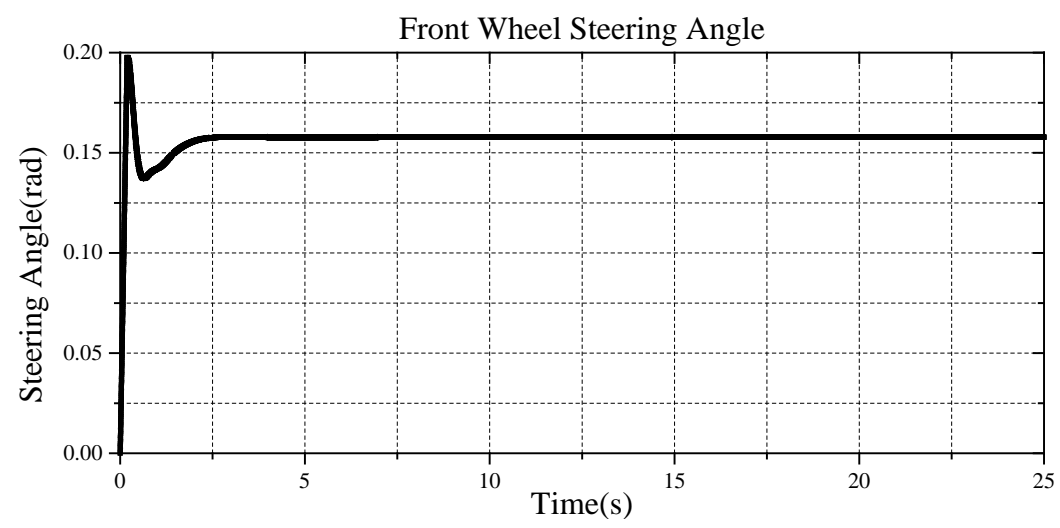

(a)

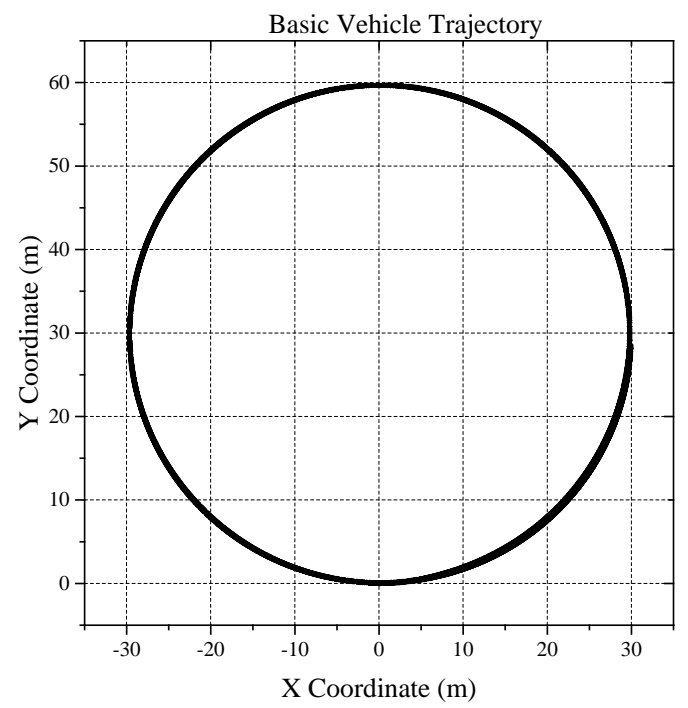

(b)

Figure 6. Simulation setup. (a) Command of front steering-wheel angle; (b) Desired neutral steering circle.

\section{Simulations and Discussion}

In this section, the simulation results of the proposed approach are given. Figure 7 shows the evaluation of the proposed EDS in Figure 3 with a different $K$ gain. Note that US stands for under-steering, OS represents over-steering, and NS is neutral-steering. Evidently, under $K=200$, the system can achieve neutral-steering, which ensures safe operation. Figure 8 shows all of the performance indexes which concern the evaluation of Figure 7. Figure 8a shows that OS is nimbler and US is more sluggish. OS is suitable for a racecar, and NS is fit for everyday drivers. Figure $8 \mathrm{~b}-\mathrm{d}$ are the longitudinal acceleration, lateral acceleration, and yaw rate of the test, respectively. To sum up, OS is nimble, NS is normal, and US is sluggish. Figure 9 shows the adjusted torque from EDS in these three scenarios. Note that, in the case of $K=500$, the vehicle falls into a state of continuous OS. At $10 \mathrm{~s}$, 
the vehicle becomes unstable as the torque of the rear left wheel increases and the torque of rear right wheel decreases.

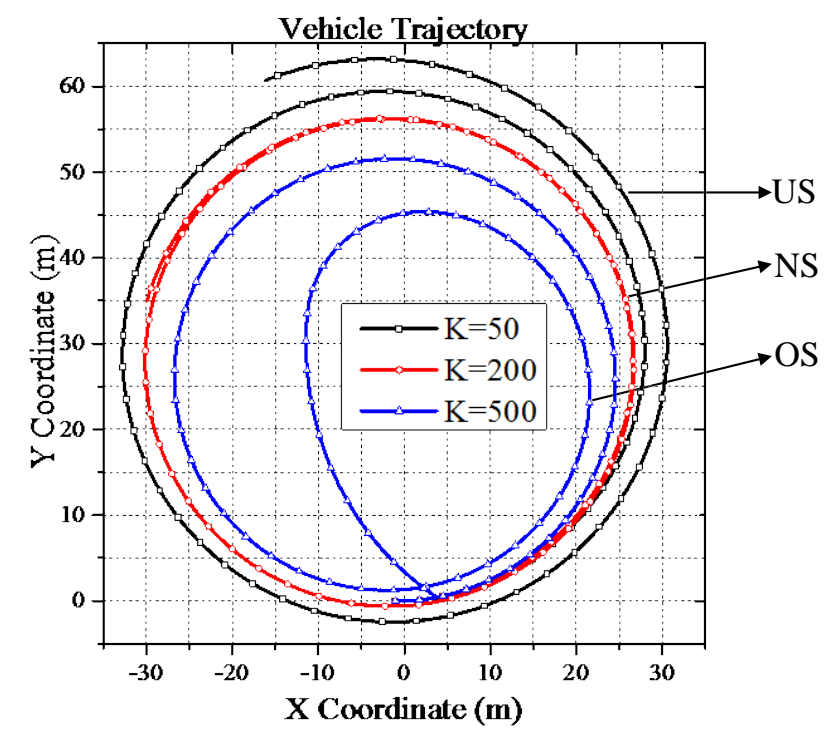

Figure 7. EDS test under different $K$ gain.

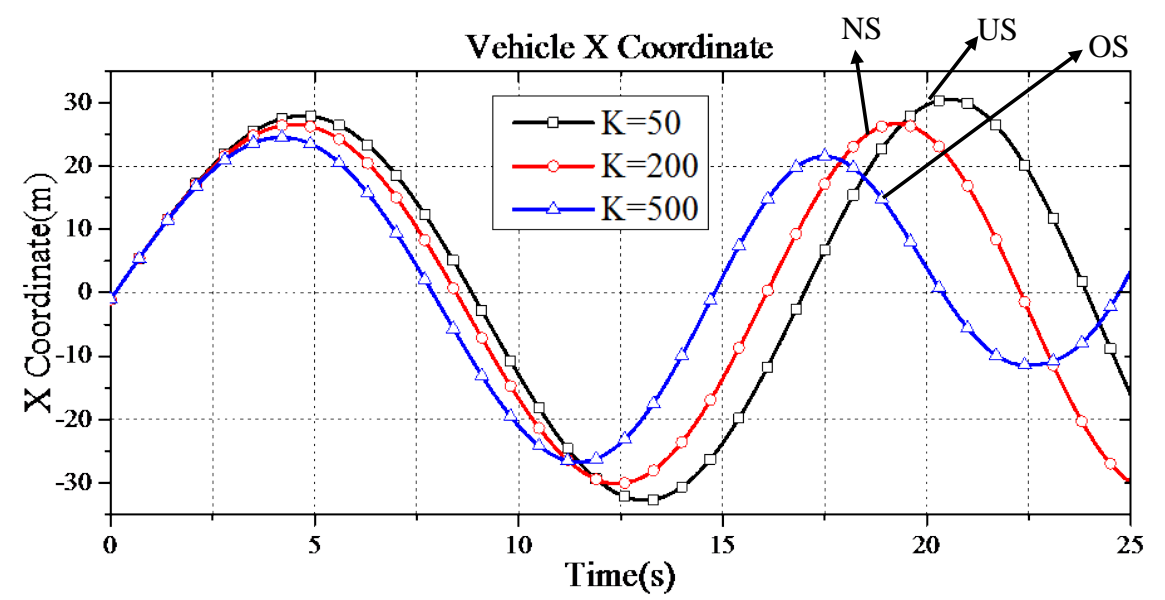

(a)

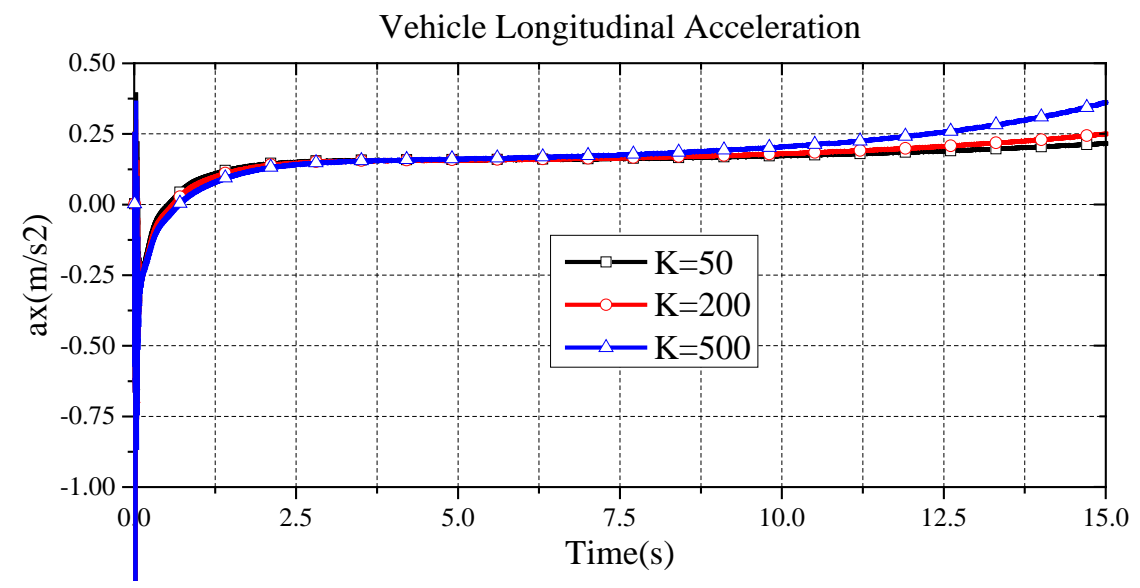

(b)

Figure 8. Cont. 


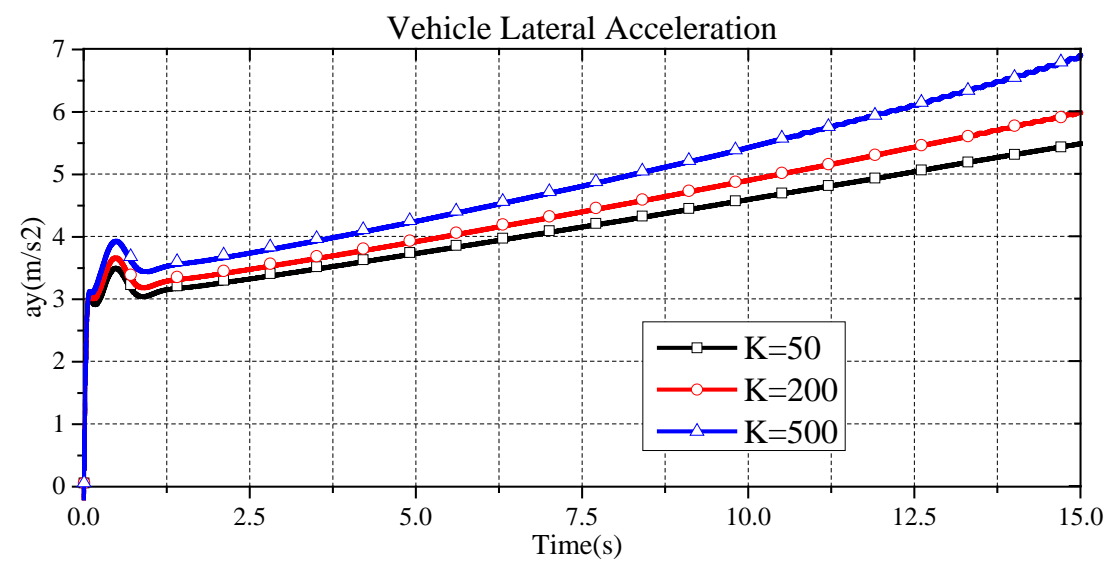

(c)

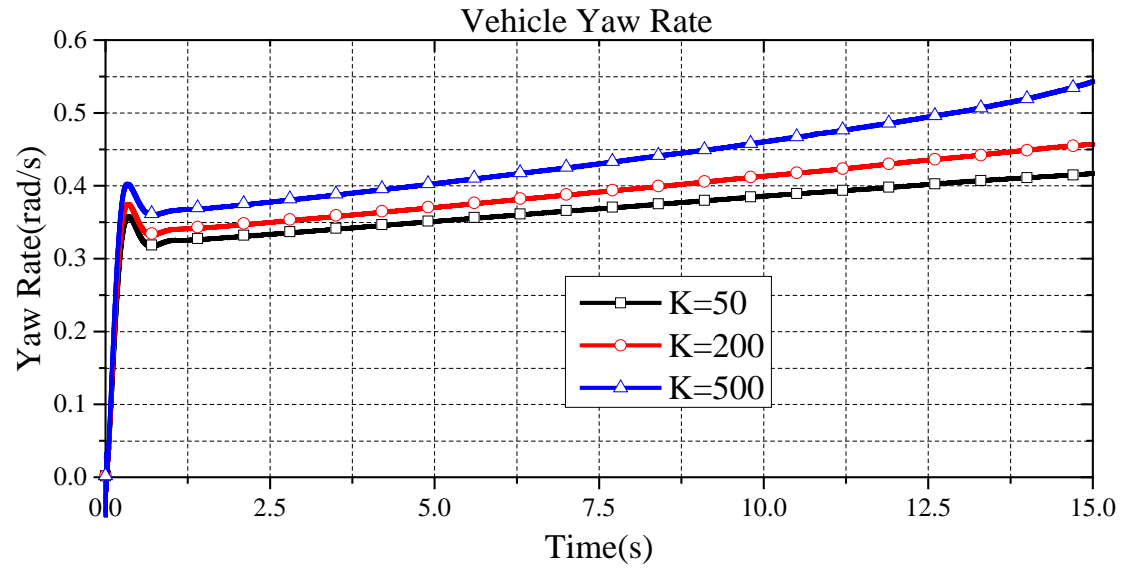

(d)

Figure 8. System performance. (a) Position of X coordinate; (b) Longitudinal acceleration; (c) Lateral acceleration; (d) Yaw rate.

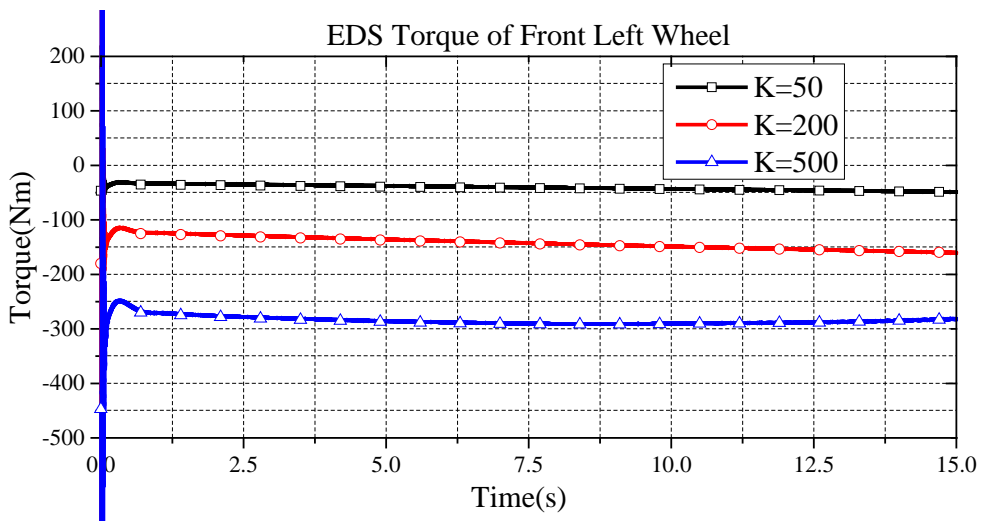

(a)

Figure 9. Cont. 


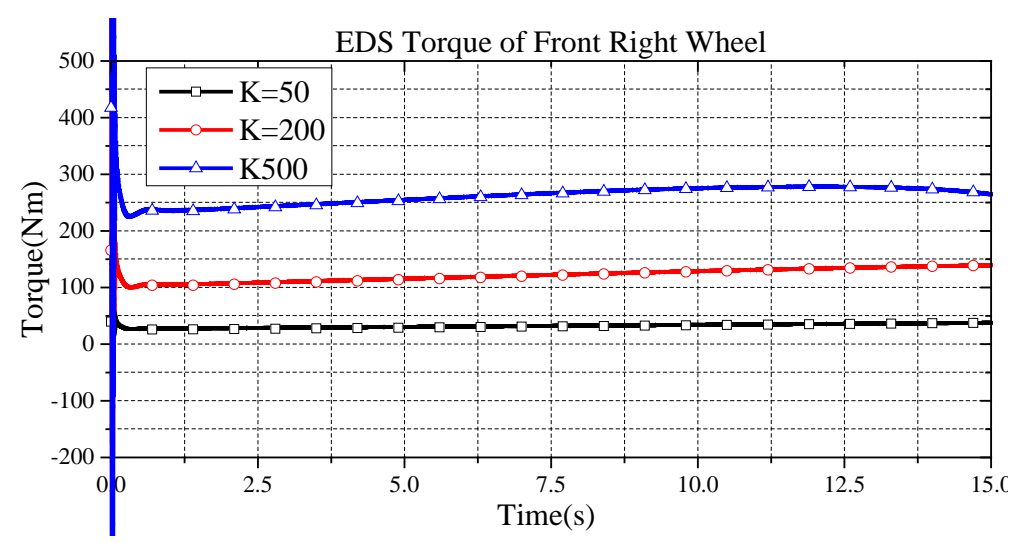

(b)

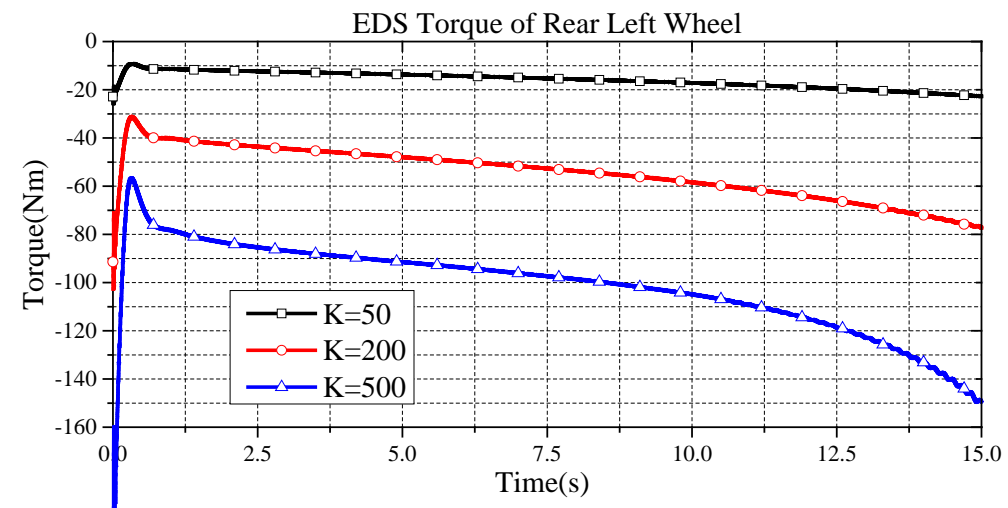

(c)

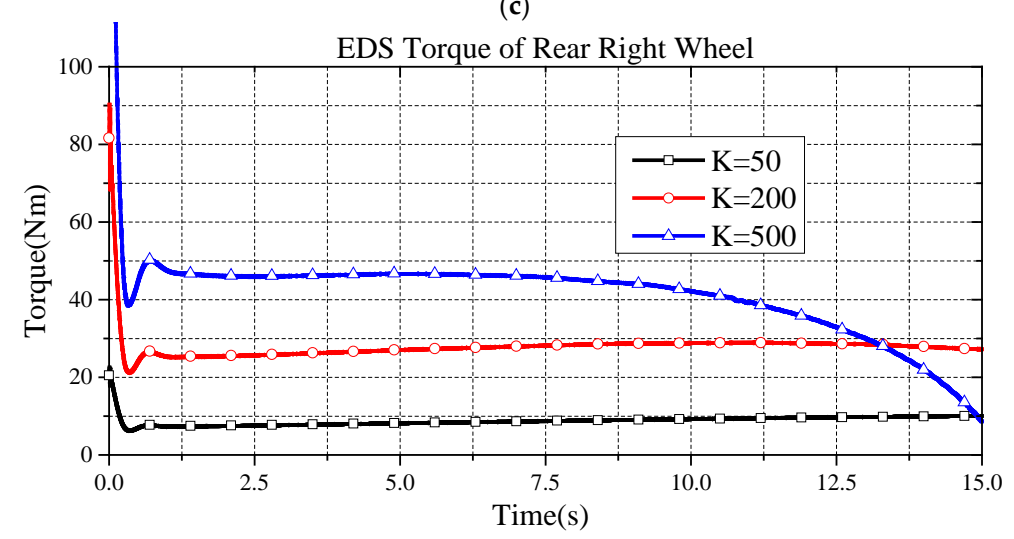

(d)

Figure 9. Adjusting torque from EDS. (a) Front left wheel; (b) Front right wheel; (c) Rear left wheel; (d) Rear right wheel.

Note that from the simulation results, if the $K$ gain is larger than $K_{\max }^{*}$, the vehicle will respond by obvious OS. This is because over-steering is based on a large speed difference between the left and right wheels. Thus, the torque difference will increase, which leads to an incensement of yaw moment and the side slip angle. It is known that the larger the side slip angle, the bigger the lateral force. However, if the lateral force exceeds its maximum value due to the saturation of the tire's physical conditions, the traction force will decay quickly according to the magic formula of tires. Clearly, because of the loss of traction force, the vehicle slides over the motion surface. Then, the vehicle motion becomes uncontrollable and thus spins. Figure 10 illustrates the analysis diagram of the gain adjusting policy. Under this discovery, adaptively adjusting the $K$ gain can easily fine-tune motion behaviors of a vehicle based on different steering modes. For example, many racecars have sport and 
urban driving modes for users to choose. Of course, different steering modes will also result in various fuel economy responses.

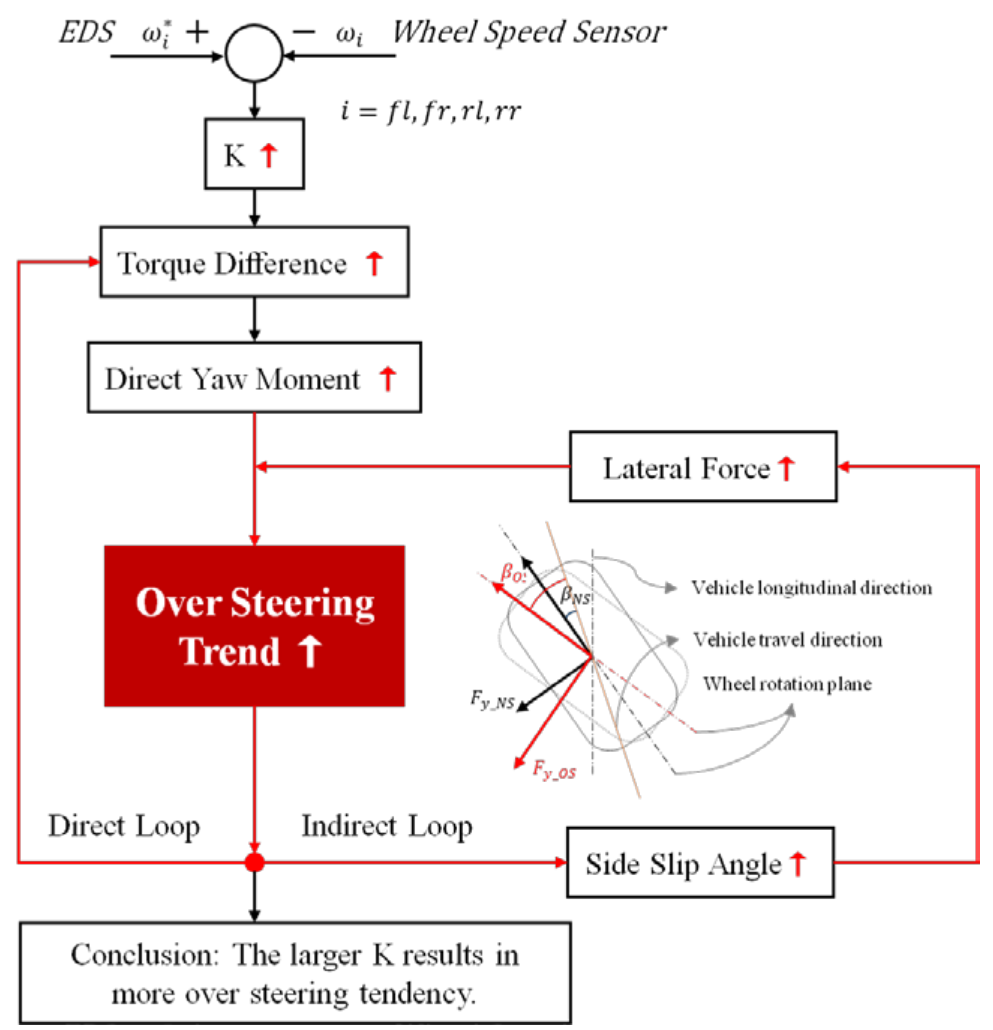

Figure 10. Analysis diagram of the gain adjusting polocy.

In addition, the performance deteriorations from the larger $K$ gain can also be found in the analysis of control theory. From the simulations, the proposed approach shows its performance on EDS. However, it also sketches some limitations which should be noted. Figure 11 shows the rearranged control block diagram of the proposed EDS. From this figure, one can obtain the transfer function as

$$
\frac{\omega_{i e}}{V^{*}}=\frac{G(s) K(s)}{1+G(s) K(s)+K G(s)-K M_{T} G(s)}
$$

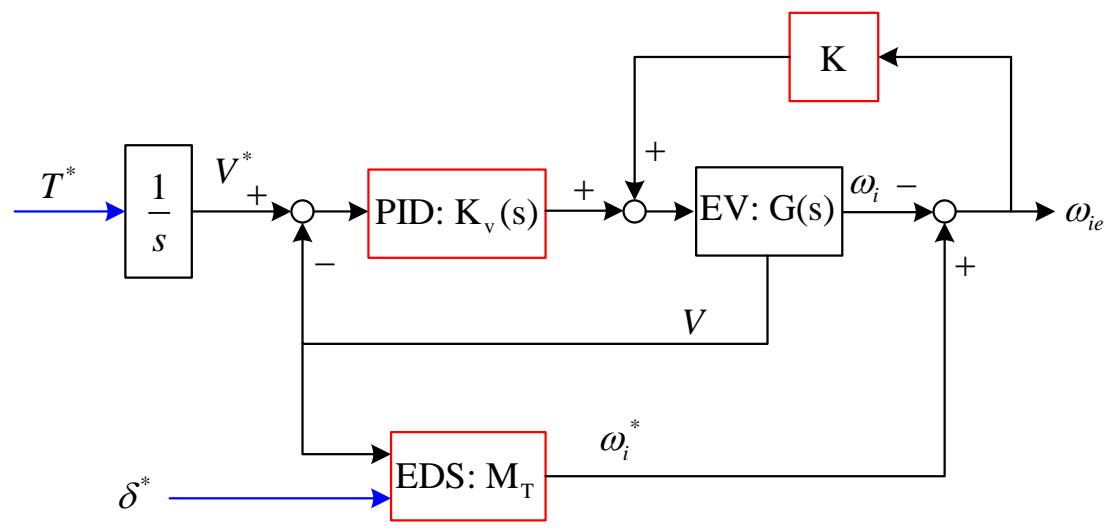

Figure 11. Control block diagram of proposed scheme. 
From Equation (8), one can observe an interesting fact that adequate $K$ gain can improve the EDS performance. However, too large of a $K$ gain will lead to an unstable scenario. The analysis concludes the same results discovered from the simulations. Consequently, by using the proposed EDS, the corresponding gain $K$ should be carefully selected.

\section{Conclusions}

This paper has established an EDS control strategy for four-wheel drive electric vehicles. Numerous steady steering simulation tests that comply with the requirements of ISO4238:2012 were carried out to study the characteristics of EDS. The simulation results demonstrated that given a larger feedback gain, the actual wheel speed can better track the reference wheel speed from the EDS. However, in this case, the vehicle shows an obvious over-steering tendency. On the contrary, the wheel speed cannot track the reference wheel speed well with a small feedback gain. In order to find the mechanism of the specific phenomenon above, this paper made a deep discussion and analysis from the aspects of vehicle dynamics and nonlinear tire property. As a conclusion, for the vehicle system with EDS control, there should be an adjustable feedback gain, rather than a fixed one, to deliver good steering characteristics. This feedback mechanism will contribute to the future research and development on EDS, especially to improvement in the vehicle steering characteristics.

Acknowledgments: This work was mainly supported by the Ministry of Science and Technology (MOST) of Taiwan, under projects MOST 104-2221-E-024-008 and MOST 105-2218-E-006-008. Part of this work was supported by "the Fundamental Research Funds for the Central Universities" of China, under projects No. 30915011305 and No. 30915118802.

Author Contributions: Dejun Yin and Danfeng Shan initiated and discussed the research problem; Jia-Sheng Hu conceived and developed the methods; Dejun Yin and Danfeng Shan performed the simulations; Jia-Sheng Hu analyzed the data; Dejun Yin and Jia-Sheng Hu prepared and wrote the paper.

Conflicts of Interest: The authors declare no conflict of interest.

\section{References}

1. Tabbache, B.; Kheloui, A.; Benbouzid, M.E.H. An adaptive electric differential for electric vehicles motion stabilization. IEEE Trans. Veh. Technol. 2011, 60, 104-110. [CrossRef]

2. Zhao, Y.E.; Zhang, J.W. Modelling and simulation of electronic differential system for an electric vehicle with two-motor-wheel drive. Int. J. Veh. Syst. Model. Test. 2009, 4, 117-131. [CrossRef]

3. Hartani, K.; Bourahla, M.; Miloud, Y.; Sekkour, M. Direct torque control of an electronic differential for electric vehicle with separate wheel drives. J. Autom. Syst. Eng. 2008, 2, 22-38.

4. Tsai, M.C.; Hu, J.S. Pilot control of an auto-balancing two-wheeled cart. Adv. Robot. 2007, 21, 817-827. [CrossRef]

5. Hu, J.-S.; Lin, X.-C.; Yin, D.; Hu, F.-R. Dynamic motion stabilization for front-wheel drive in-wheel motor electric vehicles. Adv. Mech. Eng. 2015, 7, 1-11. [CrossRef]

6. Nasri, A.; Gasbaoui, B.; Fayssal, B.M. Sliding mode control for four wheels electric vehicle drive. Procedia Technol. 2016, 22, 518-526. [CrossRef]

7. Ren, T.-J.; Chen, T.-C.; Chen, C.-J. Motion control for a two-wheeled vehicle using a self-tuning PID controller. Control Eng. Pract. 2008, 16, 365-375. [CrossRef]

8. Shimizu, H.; Harada, J.; Bland, C.; Kawakami, K.; Chan, L. Advanced concepts in electric vehicle design. IEEE Trans. Ind. Electron. 1997, 44, 14-18. [CrossRef]

9. Hori, Y. Future vehicle driven by electricity and control-research on four-wheel-motored "UOT electric march II". IEEE Trans. Ind. Electron. 2004, 51, 954-962. [CrossRef]

10. Hu, J.S.; Huang, Y.R.; Hu, F.R. Development of traction control for front-wheel drive in-wheel motor electric vehicles. Int. J. Electr. Hybrid Veh. 2012, 4, 344-358. [CrossRef]

11. ISO 4138:2012. In Passenger Cars-Steady-State Circular Driving Behaviour_Open-Loop Test Methods; International Organization for Standardization: Geneva, Switzerland, 2012. 
12. Kahveci, H.; Okumus, H.I.; Ekici, M. An electronic differential system using fuzzy logic speed controlled in-wheel brushless DC motors. In Proceedings of the 2013 Fourth International Conference on Power Engineering, Energy and Electrical Drives, Istanbul, Turkey, 13-17 May 2013.

13. Gair, S.; Cruden, A.; McDonald, J.; Hredzak, B. Electronic differential with sliding mode controller for a direct wheel drive electric vehicle. In Proceedings of the 2004 IEEE International Conference on Mechatronics, Istanbul, Turkey, 3-5 June 2004.

14. Yıldırım, M.; Öksüztepe, E.; Tanyeri, B.; Kürüm, H. Design of electronic differential system for an electric vehicle with in-wheel motor. In Proceedings of the 2016 IEEE Power and Energy Conference at Illinois (PECI), Urbana, IL, USA, 19-20 February 2016.

15. Zhou, Y.; Li, S.; Zhou, X.; Fang, Z. The control strategy of electronic differential for EV with four in-wheel motors. In Proceedings of the 2010 Chinese Control and Decision Conference, Xuzhou, China, 26-28 May 2010.

16. Hajihosseinlu, A.; Filizadeh, S.; Bistyak, G.; Dirks, E. Electronic differential design for a vehicle with four independently controlled in-wheel motors. In Proceedings of the 2014 IEEE International on Electric Vehicle Conference (IEVC), Florence, Italy, 17-19 December 2014.

17. Zhao, L.; Liu, Z. Vehicle velocity and roll angle estimation with road and friction adaptation for four-wheel independent drive electric vehicle. Math. Probl. Eng. 2014, 2014. [CrossRef]

18. Alipour, H.; Sabahi, M.; Sharifian, M.B.B. Lateral stabilization of a four wheel independent drive electric vehicle on slippery roads. Mechatronics 2015, 30, 275-285. [CrossRef]

(C) 2017 by the authors; licensee MDPI, Basel, Switzerland. This article is an open access article distributed under the terms and conditions of the Creative Commons Attribution (CC-BY) license (http://creativecommons.org/licenses/by/4.0/). 